\title{
Evaluating Polarimetric X-Band Radar Rainfall Estimators during HMT
}

\author{
SERGEY Y. MATROSOV \\ Cooperative Institute for Research in Environmental Sciences, University of Colorado, and NOAA/Earth System \\ Research Laboratory, Boulder, Colorado
}

(Manuscript received 14 April 2009, in final form 11 June 2009)

\begin{abstract}
Different relations between rainfall rate $R$ and polarimetric X-band radar measurables were evaluated using the radar, disdrometer, and rain gauge measurements conducted during the 4-month-long field experiment. The specific differential phase shift $K_{\mathrm{DP}}$-based estimators generally show less scatter resulting from variability in raindrop size distributions than with the power-based relations. These estimators depend on model assumptions about the drop aspect ratios and are not applicable for lighter rainfalls. The polynomial approximation for the mean drop aspect ratio provides $R-K_{\mathrm{DP}}$ relations that result overall in good agreement between the radar retrievals of rainfall accumulations and estimates from surface rain gauges. The accumulation data obtained from power estimators that use reflectivity $Z_{\mathrm{eh}}$ and differential reflectivity $Z_{\mathrm{DR}}$ measurements generally exhibit greater standard deviations with respect to the gauge measurements. Unlike the phase-based estimators, the power-based estimators have an advantage of being "point" measurements, thus providing continuous quantitative precipitation estimation (QPE) for the whole area of radar coverage. The uncertainty in the drop shape model can result in errors in the attenuation and differential attenuation correction procedures. These errors might provide biases of radar-derived QPE for the estimators that use power measurements. Overall, for all considered estimators, the radar-based total rainfall accumulations showed biases less than $10 \%$ (relative to gauges). The standard deviations of radar retrievals were about $23 \%$ for the mean $Z_{\mathrm{eh}}-R$ relation, $17 \%-22 \%$ for the $K_{\mathrm{DP}}$-based estimators (depending on the drop shape model), and about $20 \%-32 \%$ for different $Z_{\mathrm{eh}}-Z_{\mathrm{DR}}$-based estimators. Comparing $Z_{\mathrm{DR}}$-based retrievals of mean mass raindrop size $D_{m}$ (for $D_{m}>1 \mathrm{~mm}$ ) with disdrometer-derived values reveals an about $20 \%-25 \%$ relative standard deviation between these two types of estimates.
\end{abstract}

\section{Introduction}

The use of meteorological radars that operate at $\mathrm{X}$-band frequencies (wavelength $\lambda \sim 3 \mathrm{~cm}$ ) has been increasing over the past several years. The introduction of polarimetric methods for these radars has provided tools that help to account for power signal attenuation, which was the main limitation hindering the quantitative precipitation estimation (QPE) from using X-band radars (e.g., Matrosov et al. 2002; Anagnostou et al. 2004; Park et al. 2005). Polarimetry also provided a way to estimate rainfall rate using differential phase measurements that are immune to signal power attenuation. The stronger X-band differential phase signal, compared to the traditional radar frequencies at $\mathrm{S}$ and $\mathrm{C}$ bands (i.e., $\lambda \sim 10$ and $5 \mathrm{~cm}$, respectively), adds some additional

Corresponding author address: Sergey Y. Matrosov, R/PSD2, 325 Broadway, Boulder, CO 80305.

E-mail: sergey.matrosov@noaa.gov attractiveness to X-band radar polarimetric measurements of rainfall (Matrosov et al. 2006).

Although a typical range of X-band radar systems (usually around $40-50 \mathrm{~km}$ ) is relatively short, the lower cost, smaller sizes, and easier transportability of such radars, as compared to S- and C-band systems, make them a convenient tool for hydrometeorological studies when high temporal and spatial resolution radar coverage is needed over some limited area (e.g., Brotzge et al. 2006) and in the regions that lack adequate coverage by the National Weather Service radars (e.g., Matrosov et al. 2005). Although the concept of the X-band gapfilling radars is still debated, it is obvious that these radars are a useful tool for different applications, which require gathering rainfall parameter information at high temporal and spatial resolutions.

For a number of years, the National Oceanic and Atmospheric Administration (NOAA) in collaboration with other agencies has conducted the Hydrometeorological Testbed (HMT) West field campaigns (available

DOI: 10.1175/2009JTECHA1318.1 
online at http://hmt.noaa.gov/) in the Northern California coastal areas and the Sierra Nevada in the American River basin. Wintertime Pacific weather systems laden with moisture produce significant rainfalls in the lower terrain and snowfalls in the higher mountain area in the HMT study region. The NOAA Earth System Research Laboratory (ESRL) used its transportable polarimetric hydrometeorological X-band radar (HYDROX) during several recent HMT deployments for the purpose of mapping precipitation and estimating rainfall and snowfall parameters.

This radar, which is primarily used for the NOAA hydrometeorological studies, operates at a wavelength of $3.2 \mathrm{~cm}$. It has full scanning capability, and its technical characteristics are presented by Matrosov et al. (2005). The simultaneous transmission-simultaneous receiving (STSR) of horizontally $(h)$ and vertically $(v)$ polarized signals is used in the HYDROX radar. Although this measurement scheme is simpler than the alternative transmission schemes used by some research radars, it still allows the use of most of the polarimetric information (e.g., Doviak et al. 2000; Matrosov 2004).

Because of stronger attenuation and some deviation from Rayleigh-type scattering on raindrops, X-band radar QPE has certain distinctions from the traditional precipitation radar frequencies. A number of different rainfall parameter estimators for X-band polarimetric radar measurements have been suggested. Because all polarimetric radar signals in rainfall are caused by raindrop nonsphericity, these estimators inevitably depend on the assumed model of the drop aspect ratio as a function of drop size. The issue of the advantages and disadvantages of different X-band rainfall parameter estimators and their dependence on the drop shape model assumption is, however, still largely uncharacterized.

This paper presents a comparative evaluation of different estimators that use reflectivity, differential reflectivity, and differential phase shift measurements for retrieving rainfall rates and characteristic raindrop size from $\mathrm{X}$-band polarimetric radar measurements. This evaluation is performed using the HYDROX data measurements during the 4-month-long HMT field experiment in the 2005/06 winter season (HMT-06). This experiment provided a wide range of precipitation observations, including rainfall of different intensities. A number of surface meteorology sites were deployed in the HYDROX radar area coverage, providing the "ground truth" rainfall information.

\section{Polarimetric properties of individual drops}

Two main polarimetric radar quantities used for rainfall parameter retrievals are differential reflectivity
$Z_{\mathrm{DR}}$ and specific differential phase shift on propagation $K_{\text {DP. }}$. Depolarization ratios, which could be measured directly by radars with alternative transmission of signals with different polarization states or estimated from radars with the STSR measurement scheme (e.g., Matrosov 2004), are valuable for ice hydrometeor type and habit identification but are not widely used for quantitative rainfall retrievals. Both $K_{\mathrm{DP}}$ and $Z_{\mathrm{DR}}$ vary with raindrop shape, so polarimetric estimators of rainfall are dependent on drop shape model assumptions.

Drops that are smaller than about $0.05 \mathrm{~cm}$ in diameter are essentially spherical and do not produce polarimetric signatures which can be reliably measured. Larger drops become flattened as they fall and are usually modeled as oblate spheroids (e.g., Bringi and Chandrasekar 2001). Spheroidal aspect ratios $r$ decrease as drop sizes increase. For a number of years, a linear model that relates $r$ and equal-volume drop diameter $D_{e}$ was used by the radar community for modeling polarimetric parameters in rainfall. The linear fit to the data from Pruppacher and Beard (1970) provides a decrease of $r$ with increasing $D_{e}$ with a slope of $b=0.62 \mathrm{~cm}^{-1}$ (for $D_{e}>0.05 \mathrm{~cm}$ ). $\mathrm{X}$-band radar-based estimations of the slope $b$ in a framework of the linear model using the $Z_{\mathrm{DR}}, K_{\mathrm{DP}}$, and horizontal polarization reflectivity $Z_{\text {eh }}$ consistency approach (Gorgucci et al. 2000) provided an estimate of the mean slope $b$ of approximately $0.56 \mathrm{~cm}^{-1}$ (Matrosov et al. 2005).

Although the linear model for drop aspect ratio was used extensively in the past by the meteorological radar community, more recent studies of raindrop shapes indicate that a nonlinear polynomial approximation on average provides a better fit to the experimental data. Figure 1 shows the raindrop aspect ratios as a function of $D_{e}$ for the linear model with $b \approx 0.56 \mathrm{~cm}^{-1}$ and the polynomial approximation from Brandes et al. (2005). This approximation is also generally consistent with experimental data from Andsager et al. (1999) and Thurai and Bringi (2005). It can be seen that the linear model in Fig. 1 predicts more oblate drops than the polynomial approximation for drop sizes less than about $3 \mathrm{~mm}$ and less oblate drops for large sizes. The differences in the horizontal-to-vertical backscatter cross-sectional ratios for drops with vertical symmetry axis (i.e., $\sigma_{h} / \sigma_{v}$ in Fig. 1), however, are relatively modest and do not exceed a few tenths of $1 \mathrm{~dB}$ if expressed in logarithmic units. The backscatter cross sections were calculated for the standard center size bins of a Joss-Waldvogel disdrometer (JWD; Joss and Waldvogel 1967) using the T-matrix method (Barber and Yeh 1975), which is widely used in the radar meteorology community for modeling purposes. For a reference, Fig. 1 also shows the aspect and the backscatter cross section ratios for the equilibrium 


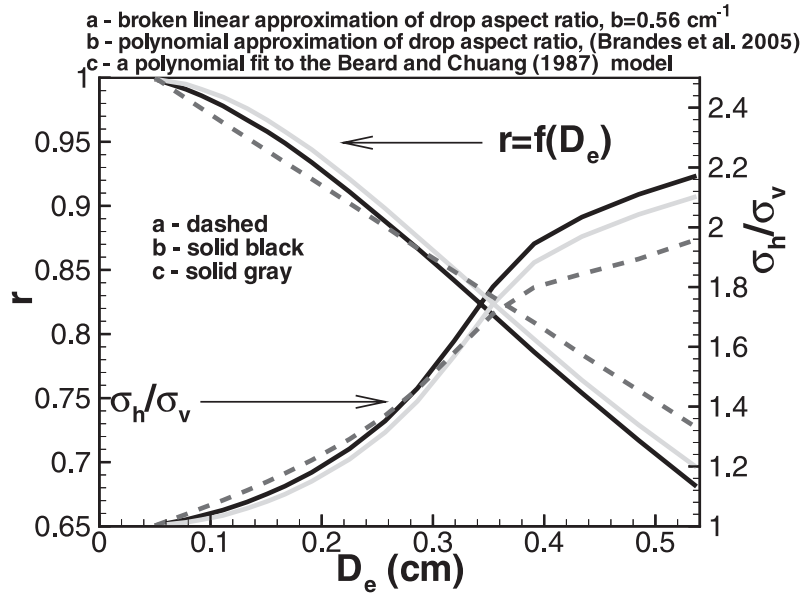

FIG. 1. Raindrop mean aspect ratio $r$ and the backscatter aspect ratio $\sigma_{h} / \sigma_{v}$ for the linear and polynomial approximations as functions of drop size. Horizontal viewing is assumed.

drop shape model from Beard and Chuang (1987). This model also has been used in polarimetric radar studies. It can be seen from Fig. 1 that the Beard and Chuang (1987) theoretical model results are in relatively close agreement with the Brandes et al. (2005) approximation of experimental data from several sources. The linear approximation (a) and the Brandes et al. (2005) polynomial approximation (b) are used further in this study.

The specific propagation differential phase shift $K_{\mathrm{DP}}$ is determined by the real part of the difference between horizontal and vertical polarization forward-scattering amplitudes $f_{h}$ and $f_{v}$. Figure 2 shows T-matrix calculations of this difference for the JWD drop sizes, assuming the different shape-size relations from Fig. 1. The calculation results were approximated by the power-law fits, which are depicted as well. It can be seen that for the linear raindrop aspect ratio model, $\operatorname{Re}\left(f_{h}-f_{v}\right)$ is proportional approximately to $D_{e}^{4.4}$, and the polynomial approximation results in $\operatorname{Re}\left(f_{h}-f_{v}\right) \sim D_{e}^{5.1}$. This implies that $K_{\mathrm{DP}}$ values for these two drop shape models are approximately proportional to 4.4th and 5.1th moments of the drop size distribution (DSD), respectively. Also shown in Fig. 2 is the product $D_{e}^{3} v\left(D_{e}\right)$, where $v\left(D_{e}\right)$ is the rain drop terminal velocity (Gunn and Kinzer 1949). This product is proportional to $D_{e}^{3.67}$ and implies a proportionality of rainfall rate $R$ to the 3.67th moment of the DSD.

The radar reflectivity factor $Z_{\mathrm{eh}}$ is proportional to the sixth moment of DSD, though there are some small deviations from this proportionality because of larger drop non-Rayleigh scattering at X band (e.g., Matrosov et al. 2006). Because there is less disparity between the DSD moments of $K_{\mathrm{DP}}$ and $R$ (compared to $Z_{\mathrm{eh}}$ and $R$ ), it is expected that $K_{\mathrm{DP}}-R$ relations should exhibit less variability resulting from DSD details than $Z_{\mathrm{eh}}-R$ re-

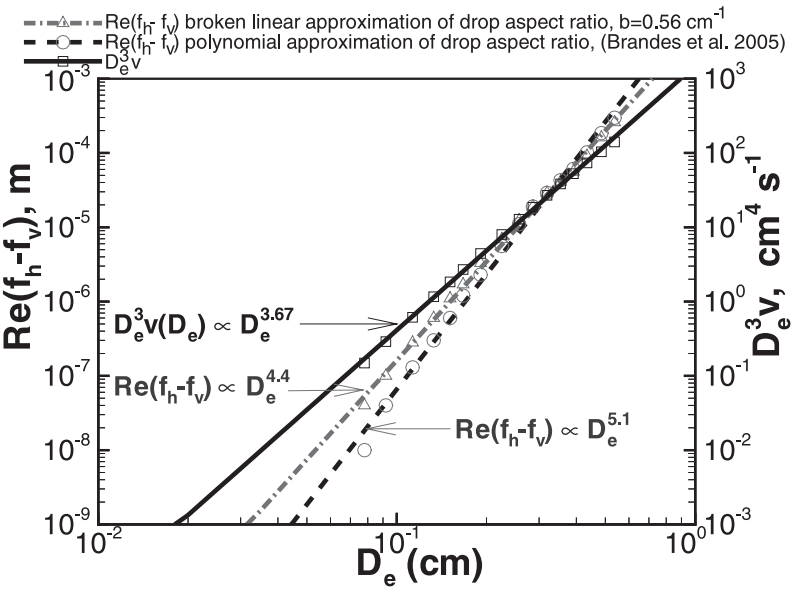

FIG. 2. Real parts of the forward-scattering amplitude differences, $\operatorname{Re}\left(f_{h}-f_{v}\right)$ as a function of raindrop size for different drop shape models.

lations. For the polynomial drop shape-size relation approximation, this $K_{\mathrm{DP}}$ advantage, however, becomes less pronounced.

\section{Rainfall parameter estimators}

\section{a. Differential phase-based estimators}

Differential phase shift-based rainfall-rate estimators have an advantage that they are not a subject to the uncertainties of the absolute calibration of the radar. This is an important factor especially for transportable radars, for which frequent set-up and tear-down procedures can result in some hardware changes that might affect the absolute radar calibration. There is an additional attractiveness of $K_{\mathrm{DP}}$ estimators at $\mathrm{X}$ band than with lower-frequency radars (e.g., S- and C-band radars), because differential phase signals are approximately proportional to the reciprocal of the wavelength (in the Rayleigh scattering regime), so X-band $K_{\mathrm{DP}}$ values are becoming usable for rainfall rates as low as about 2-3 $\mathrm{mm} \mathrm{h}^{-1}$ (Matrosov et al. 2006). Phase measurements also are not subject to attenuation effects, which present another limiting factor for power-based radar measurements at X-band.

To assess the sensitivity of $K_{\mathrm{DP}}-R$ estimators to the drop shape assumption, they were derived for the a and b drop aspect ratio models shown in Fig. 1 . The $K_{\mathrm{DP}}-R$ scatterplots are shown in Fig. 3. For data in Figs. 3a,b, they were calculated using experimental JWD DSD measurements in HMT-06 according to

$K_{\mathrm{DP}}=\left(\frac{180}{\pi}\right) \lambda \sum_{i} \operatorname{Re}\left[f_{h}\left(D_{\mathrm{ei}}\right)-f_{v}\left(D_{\mathrm{ei}}\right)\right] n_{i}\left(D_{\mathrm{ei}}\right) \quad$ and 
HMT-06, polynomial approximation for drop shapes

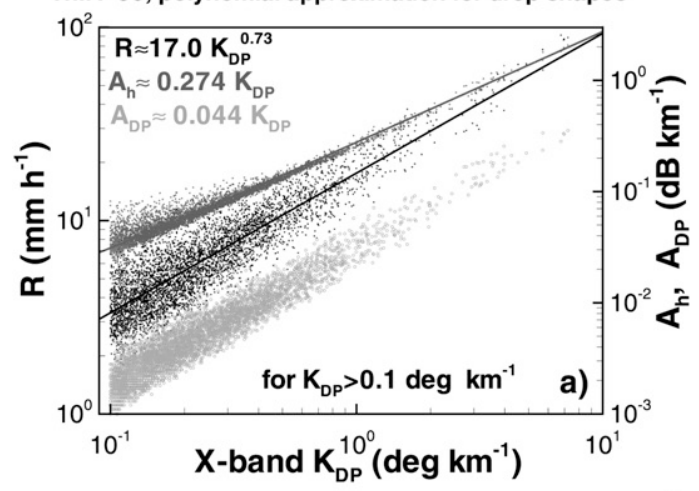

HMT-06, linear approximation for drop shapes, $b=0.56 \mathrm{~cm}^{-1}$

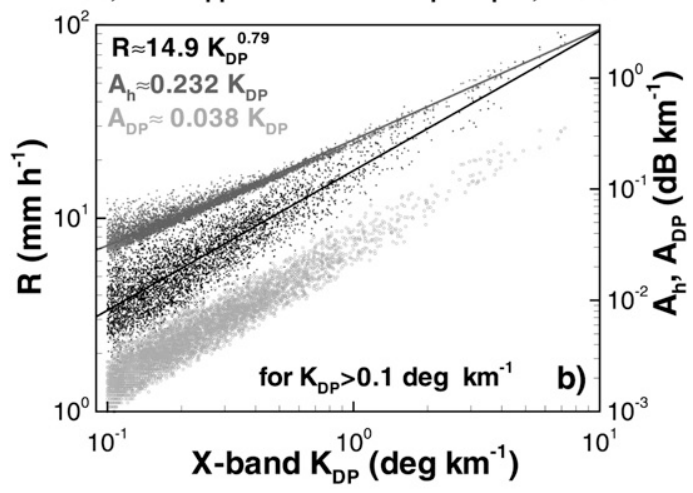

HMT-07, polynomial approximation for drop shapes

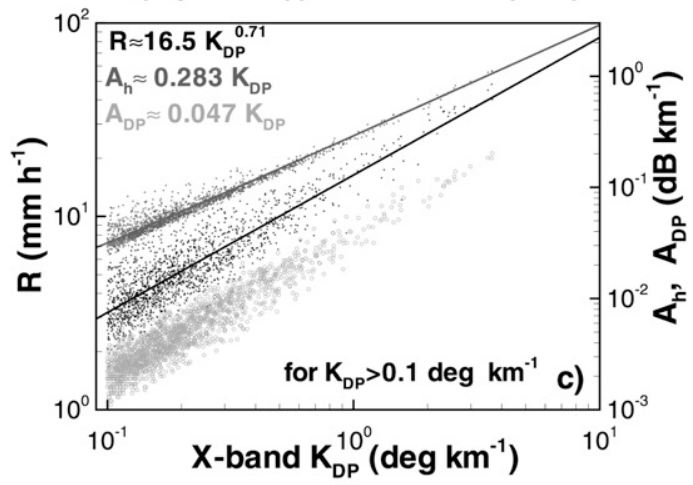

HMT-07, linear approximation for drop shapes, $b=0.56 \mathrm{~cm}^{-1}$

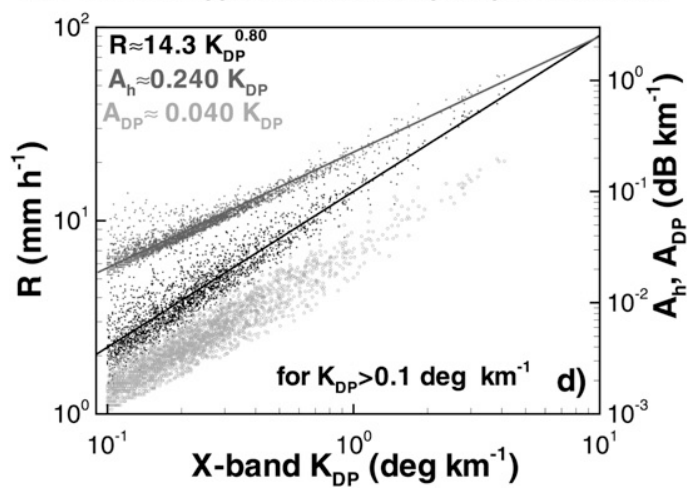

FIG. 3. $R-K_{\mathrm{DP}}$ (black), $A_{h}-K_{\mathrm{DP}}$ (dark gray), and $A_{\mathrm{DP}}-K_{\mathrm{DP}}$ (light gray) scatterplots for (a),(b) HMT-06 and (c),(d) HMT-07 DSDs for the (a),(c) polynomial and (b),(d) linear drop aspect ratio models.

$$
R=\sum_{i}\left(\frac{\pi}{6}\right) D_{\mathrm{ei}}^{3} n_{i}\left(D_{\mathrm{ei}}\right) \nu_{i}\left(D_{\mathrm{ei}}\right),
$$

where $f_{h}$ and $f_{v}$ are the frequency-dependent scattering amplitudes defined earlier and $n_{i}$ represents raindrop concentrations from 20 size bins $\left(D_{\text {ei }}\right)$ measured by a JWD deployed at the Colfax (CFC) ground validation site, which was located $18.3 \mathrm{~km}$ from the radar during the HMT-06 field deployment from December 2005 to March 2006. Concentration values were corrected for the "dead time" effects, according to Sheppard and Joe (1994). Also depicted in Fig. 3 are scatterplots of horizontal polarization attenuation coefficient $A_{h}$ and differential attenuation coefficient $A_{\mathrm{DP}}$. The relations between $A_{h}$ and $K_{\mathrm{DP}}$ and between $A_{\mathrm{DP}}$ and $K_{\mathrm{DP}}$ are essential for correcting reflectivity and differential reflectivity measurements for attenuation (for $Z_{\mathrm{eh}}$ ) and differential attenuation (for $Z_{\mathrm{DR}}$ ). The $A_{h}$ and $A_{\mathrm{DP}}$ values were calculated according to

$$
\begin{aligned}
A_{h} & =8.68 \lambda \sum_{i} \operatorname{Im} f_{h}\left(D_{\text {ei }}\right) n_{i}\left(D_{\text {ei }}\right) \quad \text { and } \\
A_{\mathrm{DP}} & =8.68 \lambda \sum_{i} \operatorname{Im}\left[f_{h}\left(D_{\mathrm{ei}}\right)-f_{v}\left(D_{\mathrm{ei}}\right)\right] n_{i}\left(D_{\mathrm{ei}}\right),
\end{aligned}
$$

where the attenuation coefficients are in decibels per unit length. An assumption of the mean temperature of $6^{\circ} \mathrm{C}$ was made during calculations with (3) and (4). Temperature measurements in the vicinity of the HYDROX radar deployment during HMT-06 events were generally between $12^{\circ}$ and $4^{\circ} \mathrm{C}$, so this assumption is believed to be representative of the mean conditions.

The best power-law fits for the $R-K_{\mathrm{DP}}$ relations and best linear fits for $A_{h}-K_{\mathrm{DP}}$ and $A_{\mathrm{DP}}-K_{\mathrm{DP}}$ relations for the HMT-06 DSDs are also shown in Figs. 3a,b. For the same $K_{\mathrm{DP}}$ value, polynomial drop shape relation (i.e., $R=17.0 K_{\mathrm{DP}}^{0.73}$ ) provides generally higher rainfall rates (except for very large values of $K_{\mathrm{DP}}$ ) than the relation with a linear drop shape (i.e., $R=14.9 K_{\mathrm{DP}}^{0.79}$ ). This is because, for drops smaller than about $3 \mathrm{~mm}$, the polynomial shape-size model prescribes less oblate shapes than the linear shape-size model (see Fig. 1). The difference between results of the two $R-K_{\mathrm{DP}}$ relations is higher for lower $K_{\mathrm{DP}}$ values (e.g., around $30 \%$ for $K_{\mathrm{DP}} \sim$ $0.1^{\circ} \mathrm{km}^{-1}$ ), although it diminishes as rainfall becomes heavier.

The exponent in the $R-K_{\mathrm{DP}}$ relation is a little smaller for the polynomial drop shape model, and this relation provides slightly greater data scatter with respect to the best-fit line [the corresponding relative standard deviation (SD) is about $27 \%$ versus $22 \%$ for the linear drop shape model]. Note that these SD values are noticeably smaller than the standard deviation for the 


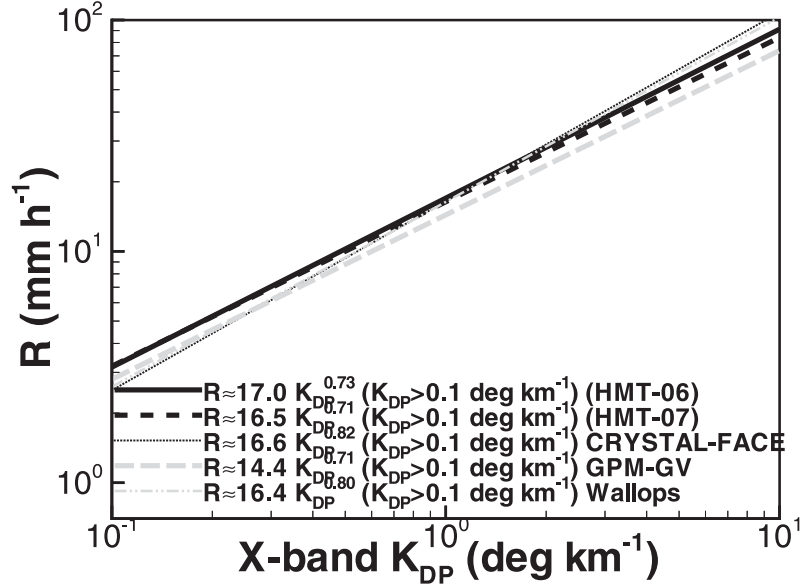

FIG. 4. $R-K_{\mathrm{DP}}$ power-law fit relations obtained with DSDs from different field campaigns.

HMT-06 $Z_{\mathrm{eh}}-R$ relation $\left(Z_{\mathrm{eh}}=100 R^{1.76}\right)$, which is about $40 \%$ (Matrosov et al. 2007). This reflects more direct relations between $K_{\mathrm{DP}}$ and $R$ than between reflectivity and $R$, as discussed earlier. The uncertainty in the raindrop shape model, however, diminishes this advantage of differential phase shift-based estimates. Accounting for the drop canting will increase the coefficients in relations shown in Fig. 3. For the zero mean canting angle and a typical canting angle standard deviation of $8^{\circ}$, the corresponding increase is about $4 \%$ (Matrosov et al. 2002).

The polynomial raindrop shape model also provides higher coefficients in attenuation-differential attenuation correction relations (i.e., 0.274 and $0.044 \mathrm{~dB} \mathrm{deg}^{-1}$ versus 0.232 and $0.038 \mathrm{~dB} \mathrm{deg}^{-1}$ for the linear drop shape model). The uncertainty in the drop shape model would result in the uncertainties of the HYDROX attenuationdifferential attenuation correction procedures, which are described by Matrosov et al. (2005). If the whole area of the HYDROX radar coverage in HMT-06 $(\sim 38 \mathrm{~km})$ were filled with rain of about $9 \mathrm{~mm} \mathrm{~h}^{-1}$ (which is about the heaviest mean rain rate observed during HMT-06), the correction result differences resulting from the shape-size model (linear versus polynomial) would be about $1.2 \mathrm{~dB}$ for $Z_{\mathrm{eh}}$ and $0.2 \mathrm{~dB}$ for $Z_{\mathrm{DR}}$. These values are on the order of the measurement-calibration uncertainties of the radar. Note also that temperature variability may also add some uncertainties in the correction procedures. For HMT-06 conditions, these uncertainties are likely to be less than $1 \mathrm{~dB}$.

For a given raindrop shape model, there is relatively modest variability in mean $R-K_{\mathrm{DP}}$ relations from different DSD datasets. This is illustrated in Fig. 4, where the best-fit power-law $R-K_{\mathrm{DP}}$ approximations calculated for a number of JWD DSD datasets using the polynomial drop shape model are shown. These datasets were ob- tained in different geographical areas and comprise quite different rainfalls. They include data collected during the Wallops field experiment (Matrosov et al. 2002), Cirrus Regional Study of Tropical Anvils and Cirrus Layers Florida Area Cirrus Experiment (CRYSTAL-FACE), Global Precipitation Mission-Ground Validation (GPMGV) pilot study (Matrosov et al. 2006), and HMT-07. Overall, the relative variability of $R-K_{\mathrm{DP}}$ relations among different datasets is smaller than that for $Z_{\mathrm{eh}}-R$ relations.

Especially close are the $R-K_{\mathrm{DP}}$ relations from HMT-06 and HMT-07 DSDs. This is true also for other considered relations (i.e., $A_{h}-K_{\mathrm{DP}}$ and $A_{\mathrm{DP}}-K_{\mathrm{DP}}$ relations). It can be seen from comparing data in Figs. $3 \mathrm{a}$, b with data in Figs. 3c,d, where results from HMT-07 DSDs are shown (note that the HYDROX radar was not deployed during the HMT-07 project). The good correspondence between HMT-06 and HMT-07 relations suggests that DSDs in the HMT West area studies during the wet winter season might change relatively little on average.

\section{b. Differential reflectivity-based estimators for characteristic raindrop sizes}

Differential reflectivity $Z_{\mathrm{DR}}$ measurements provide a means for estimating characteristic drop size. Massweighted equivalent drop diameter $D_{m}$ is one of such sizes characterizing a whole DSD; $D_{m}$ is very close to the median volume drop diameter $D_{0}$ (typically within $10 \%$ ), and it can be easily estimated from JWD data. The $D_{m}-Z_{\mathrm{DR}}$ relations are typically sought in a powerlaw form (e.g., Bringi and Chandrasekar 2001). Figure 5 shows $D_{m}-Z_{\mathrm{DR}}$ scatterplots for linear and polynomial drop shape models calculated from HMT-06 DSDs using the following sums:

$$
\begin{aligned}
D_{m} & =\sum_{i} D_{\mathrm{ei}}^{4} n_{i}\left(D_{\mathrm{ei}}\right)\left[\sum D_{\mathrm{ei}}^{3} n_{i}\left(D_{\mathrm{ei}}\right)\right]^{-1}, \\
Z_{\mathrm{ep}} & =\lambda^{4} \pi^{-5}\left|\frac{\left(m_{w}^{2}+2\right)}{\left(m_{w}^{2}-1\right)}\right|^{2} \sum_{i} \sigma_{p}(D) n_{i}\left(D_{\mathrm{ei}}\right), \quad \text { and } \\
Z_{\mathrm{DR}} & =10 \log _{10}\left(\frac{Z_{\mathrm{eh}}}{Z_{\mathrm{ev}}}\right),
\end{aligned}
$$

where the subscript $p$ is either horizontal $h$ or vertical $v$ and $m_{w}$ is the complex refractive index of water.

The best-fit power-law approximations are also shown in Fig. 5. It can be seen that for larger drop populations (e.g., $Z_{\mathrm{DR}} \sim 2 \mathrm{~dB}$ ), both drop shape models provide similar $D_{m}$ results; however, for DSDs consisting of smaller drops (e.g., $Z_{\mathrm{DR}} \sim 0.2 \mathrm{~dB}$ ), the use of the linear drop shape model underestimates characteristic drop sizes by as much as $20 \%$. The average data scatter (in terms of the standard deviation with respect to the best fit) for both models is about $15 \%$. It should be mentioned 

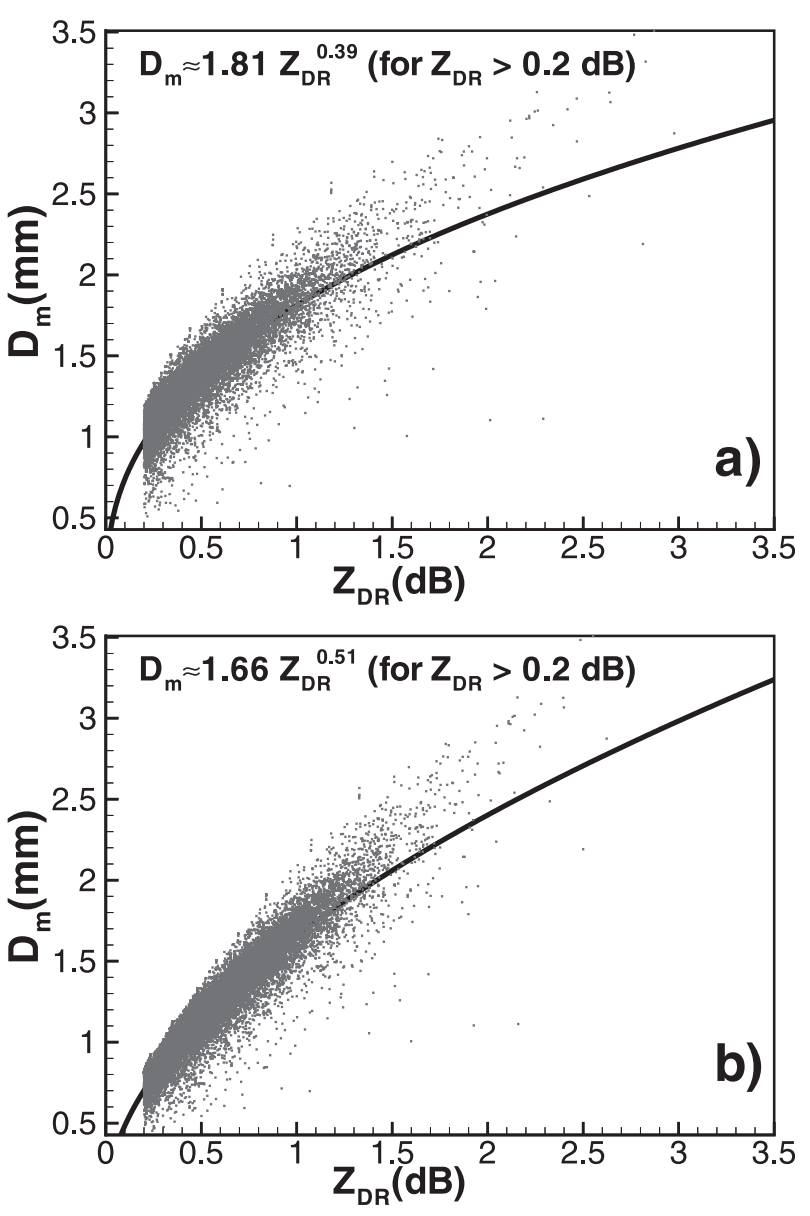

FIG. 5. $D_{m}-Z_{\mathrm{DR}}$ scatterplots for (a) polynomial and (b) linear drop shape models, as calculated for HMT-06 DSDs.

that, for canted drops, polarization crosstalk contaminates somewhat differential reflectivity values if measurements are performed in the STSR mode. For typical drop canting $\left(\sim 10^{\circ}\right)$, the corresponding deviations from the "true" $Z_{\mathrm{DR}}$ in the alternative polarization transmission scheme, however, are expected to be less than a few tenths of $1 \mathrm{~dB}$ at a $Z_{\mathrm{DR}}=2 \mathrm{~dB}$ level and diminishing with $Z_{\mathrm{DR}}$ (Matrosov et al. 2002).

\section{c. $Z_{e h}-Z_{D R}$ estimators for rainfall rate}

Although, for the given drop shape model, $K_{\mathrm{DP}}$-based rainfall estimators have advantages of being immune to the radar absolute calibration and exhibiting lower variability to the DSD details (compared to traditional $Z_{\mathrm{eh}}-R$ relations), their drawbacks are noisiness of $K_{\mathrm{DP}}$ values and some dependence on the procedure of $K_{\mathrm{DP}}$ calculations, which are computed as a range derivative of the differential phase measured by the radar. As a result, $K_{\mathrm{DP}}$-based estimates of rainfall rate are representative of some range interval $\Delta h$ centered at a given range gate ( $\Delta h$ is typically a few kilometers), unlike $Z_{\mathrm{eh}^{-}}$and $Z_{\mathrm{DR}^{-}}$ based estimates, which are referred to the given radar range gate. Because of this, the maximum radar range for differential phase-based estimates of rainfall is reduced by $\Delta h / 2$, and these estimates are not available at ranges closer than $\Delta h / 2$ either. Besides, $K_{\mathrm{DP}}$ values are too noisy for lighter rainfall and can only be used (with $\mathrm{X}$-band radars) for rainfalls, which result in radar reflectivities greater than about 26-30 dBZ (Matrosov et al. 2006). Backscatter differential phase shift can also provide additional problems for $K_{\mathrm{DP}}$-based estimates of rainfall at $\mathrm{X}$ band, although the experience from field experiment data collected with the HYDROX radar does not indicate that it is a significant problem (at least in stratiform-like rainfalls).

The combination of $Z_{\mathrm{eh}}$ and $Z_{\mathrm{DR}}$ measurements allows the use of polarimetric information while overcoming some of the $K_{\mathrm{DP}}$ problems mentioned earlier. Although differential reflectivity data are usually noisier than single polarization reflectivity, they are (unlike $K_{\mathrm{DP}}$ ) "point" measurements. $Z_{\mathrm{eh}}-Z_{\mathrm{DR}}$ rainfall-rate estimators are customary sought in the power-law form (i.e., $R=c Z_{\mathrm{eh}}^{\alpha} Z_{\mathrm{dr}}^{\beta}$ ). Bringi and Chandrasekar (2001) suggested the following estimator for a $10-\mathrm{GHz}$ frequency:

$$
R=0.0039 Z_{\mathrm{eh}}^{1.07} Z_{\mathrm{dr}}^{-5.97},
$$

where $Z_{\mathrm{dr}}$ is expressed in a linear scale [i.e., $Z_{\mathrm{DR}}=$ $\left.10 \log _{10}\left(Z_{\mathrm{dr}}\right)\right], R$ is in $\mathrm{mm}^{-1}$, and $Z_{\text {eh }}$ is in $\mathrm{mm}^{6} \mathrm{~m}^{-3}$. These authors also mentioned that, if characteristic raindrop size does not vary, $R$ and $Z_{\text {eh }}$ should be approximately proportional. Because $Z_{\mathrm{DR}}$ can be considered as a proxy for such size, it is expected that the exponent $\alpha$ in $Z_{\mathrm{eh}}-Z_{\mathrm{DR}}$ power-law estimators should be around 1. The Beard and Chuang (1987) drop shape model and a wide variety of DSDs were used for deriving (8). Although the HYDROX radar frequency is slightly different (i.e., $9.375 \mathrm{GHz}$ ), the small frequency difference should not be too critical, especially for light and moderate rainfalls. The estimator (8) has been used by the radar meteorology community, so it is instructive to use it also with the HMT-06 HYDROX radar data. In addition to (8), a power-law $Z_{\mathrm{eh}}-Z_{\mathrm{DR}}$ estimator was also derived using the HMT-06 DSDs and the polynomial drop shape-size model. This derivation yielded the following values of the parameters in this estimator: $c=$ $0.0056, \alpha=1.02$, and $\beta=-5.6$, which are not very different from the generic estimator (8). The HMT-06 tuned $Z_{\mathrm{eh}}-Z_{\mathrm{DR}}$ estimator was also applied to the HYDROX radar measurements.

Another consideration can be proposed for suggesting $Z_{\mathrm{eh}}-Z_{\mathrm{DR}}$ rainfall-rate estimators. It was shown previously (e.g., Gorgucci et al. 1992, 2006; Goddard et al. $1994)$ that, for a given drop shape model, $Z_{\mathrm{eh}}, Z_{\mathrm{DR}}$, and 

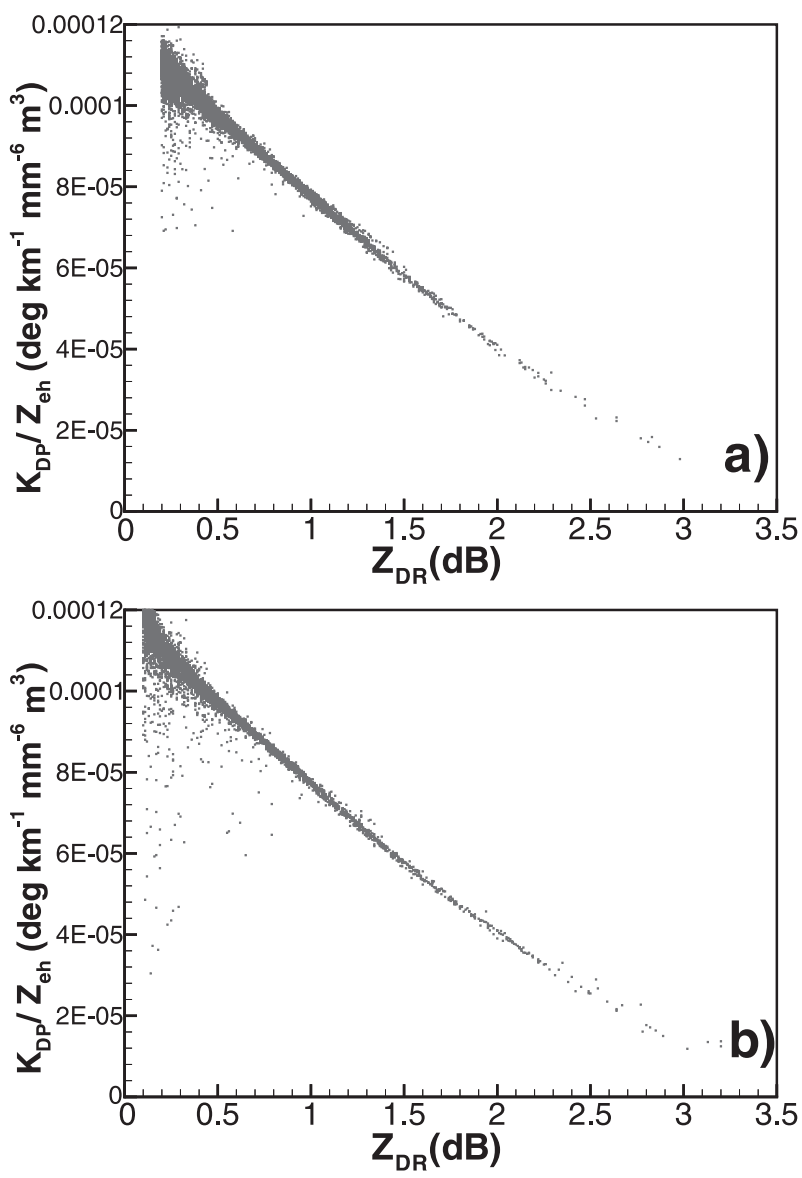

FIG. 6. $K_{\mathrm{DP}}-\left(Z_{\mathrm{eh}}-Z_{\mathrm{DR}}\right)$ consistency relations as calculated from (a) HMT-06 and (b) HMT-07 DSD data for the polynomial drop shape model.

$K_{\mathrm{DP}}$ values are generally redundant. This is the so-called self-consistency principle, and it potentially allows calculations of any parameter of these three if the other two are known. For the polynomial raindrop shape assumption, Fig. 6 shows the ratio of $K_{\mathrm{DP}} / Z_{\mathrm{eh}}$ as a function of $Z_{\mathrm{DR}}$ as calculated for HMT DSDs measured by the Joss-Waldvogel disdrometer. It can be seen that, although there is some variability resulting from the details of DSDs (especially for $Z_{\mathrm{DR}}$ values that are lower than $0.5 \mathrm{~dB}$ ), the data points for each DSD are more or less grouped along a distinct $K_{\mathrm{DP}} / Z_{\mathrm{eh}}-Z_{\mathrm{DR}}$ relation. There is not much difference between these relations for HMT-06 and HMT-07 DSDs. Although the $K_{\mathrm{DP}} / Z_{\mathrm{eh}^{-}}$ $Z_{\mathrm{DR}}$ dependence is generally nonlinear, the linearity approximately holds for $Z_{\mathrm{DR}}<1.6 \mathrm{~dB}$. Because there are relatively few data points with $Z_{\mathrm{DR}}>1.6 \mathrm{~dB}$ (at least in the HMT datasets, as seen in Fig. 6), the following approximation can be suggested for the X-band:

$$
K_{\mathrm{DP}}=Z_{\mathrm{eh}}\left(0.00012-0.000041 Z_{\mathrm{DR}}\right),
$$

where $K_{\mathrm{DP}}, Z_{\mathrm{eh}}$, and $Z_{\mathrm{DR}}$ are in ${ }^{\circ} \mathrm{km}^{-1}, \mathrm{~mm}^{6} \mathrm{~m}^{-3}$, and $\mathrm{dB}$, respectively.

If (9) is then substituted in the best-fit power-law HMT-06 $R-K_{\mathrm{DP}}$ relation, the following estimator can be obtained for the polynomial drop shape model assumption from Brandes et al. (2005):

$$
R=17\left[Z_{\mathrm{eh}}\left(0.00012-0.000041 Z_{\mathrm{DR}}\right)\right]^{0.73} .
$$

This drop shape model was chosen because recent experimental studies (Thurai and Bringi 2005) indicate that this drop shape assumption provides a good approximation to mean drop shapes and it is increasingly used within the polarimetric radar community. Note also that the best-fit $K_{\mathrm{DP}}-R$ relations for HMT-06 and HMT-07 are rather close (see Figs. 3, 4), so the estimator (10) would require only modest coefficient tuning for use in HMT-07.

Unlike the $R-K_{\mathrm{DP}}$ relation, the polarimetric consistency $Z_{\mathrm{eh}}-Z_{\mathrm{DR}}-$ based relation is a point estimator, and the corresponding retrievals are available at every radar range gate. It can be used for rainfall of any intensity, thus overcoming another important limitation of the $K_{\mathrm{DP}}$-based retrievals, which are generally not available for rainfall lighter than $2-3 \mathrm{~mm} \mathrm{~h}^{-1}$. With point measurements, an uncertainty resulting from the choice of the differential phase range-estimation interval $\Delta h$ is also avoided. The consistency estimators, however, depend on absolute reflectivity and differential reflectivity calibrations and are subject to uncertainties introduced by the attenuation-differential attenuation corrections (though these uncertainties, as will be shown later, are reduced compared to traditional $Z_{\mathrm{eh}}-R$ estimators). It should be mentioned also that, for polarimetric consistency $Z_{\mathrm{eh}}-Z_{\mathrm{DR}}$ rainfall estimators, $R$ for a constant $Z_{\mathrm{DR}}$ is proportional to a lower power of reflectivity (about $0.71-0.8$, according to the relations from different field campaigns shown in Fig. 4) than the traditional powerlaw $Z_{\mathrm{eh}}-Z_{\mathrm{DR}}$-based rainfall estimators in the form (8), which is, in some way, counterintuitive to the expectation that, for a given $Z_{\mathrm{DR}}$ value (i.e., a proxy of the characteristic drop size), $R$ and $Z_{\text {eh }}$ should be approximately proportional.

\section{Comparisons of radar-derived rainfall with surface measurements during HMT-06}

The HMT-06 field project was held during December 2005-March 2006 in the North Fork of the American River basin. The HYDROX radar was deployed near the city of Auburn, California, and was scanning at the elevation angle of $3^{\circ}$ in the direction of the sloping terrain of the Sierra Nevada, with a $150-\mathrm{m}$ gate resolution. This radar was prior calibrated using the corner 
reflector measurements. The absolute reflectivity calibrations were then verified by comparing radar measurements over a disdrometer (after correcting for attenuation effects) with $Z_{e}$ values derived from disdrometer DSDs. The $Z_{\mathrm{DR}}$ offset was calibrated using the vertical beam measurements, and $K_{\text {DP }}$ values were estimated using a "sliding window" interval containing 21 resolution gates.

The 2005/06 winter season in central California was wetter than usual. A total of 14 intensive operation periods (IOPs) from early December to late March were conducted during the HMT-06 deployment. The HYDROX radar operated during 12 rainfall events. The meteorological conditions varied from relatively warm, when the radar beam was in the rain region for up to the maximum radar range $(\sim 38.4 \mathrm{~km})$, to the cold cases, when the radar observed mostly melting layer and snow regions. The rain was mostly of the stratiform type, with distinct reflectivity and differential reflectivity brightband signatures. The copolar correlation coefficient $\rho_{\text {hv }}$ provided the most robust separation of rain from melting hydrometeors (Matrosov et al. 2007). Four surface meteorology sites, equipped with calibrated tipping-bucket (TB) rain gauges, which have manufacturer-reported accuracies better than $2 \%$ (e.g., Campbell Scientific 2008), were deployed in the area of the HYDROX radar coverage. The CFC site was also equipped with the JWD. The time periods when the radar observed rain were used for testing the rainfall parameter estimators discussed in section 3 .

\section{a. Comparisons of total rainfall accumulations}

Because of spatial variability of rainfall, beam pointing issues, and vastly different radar and direct sensor sample sizes, direct comparisons of instantaneous rainfall rates derived from radar measurements and from validation data provided by gauges and disdrometers are not very robust and present some uncertainty. Comparing rainfall accumulations may be preferable, because time-integrated parameters (e.g., rainfall accumulation) exhibit less spatial variability, which makes the influence of vastly different sampling sizes relatively less important.

IOP 4, observed on 30-31 December 2005, is of special interest. It was an extreme California winter storm event, which raised river flows to dangerous levels and produced significant flooding in many areas. Approximately $200 \mathrm{~mm}$ of rainfall accumulation in less than $24 \mathrm{~h}$ was observed in the American River basin. For the ground validation sites at CFC and Forest Hill (FHL) located at approximately 18.3 and $25.7 \mathrm{~km}$ from the radar, respectively, Fig. 7 shows the time series of rainfall accumulation as calculated from the estimators discussed earlier (using the radar data at the closest gates
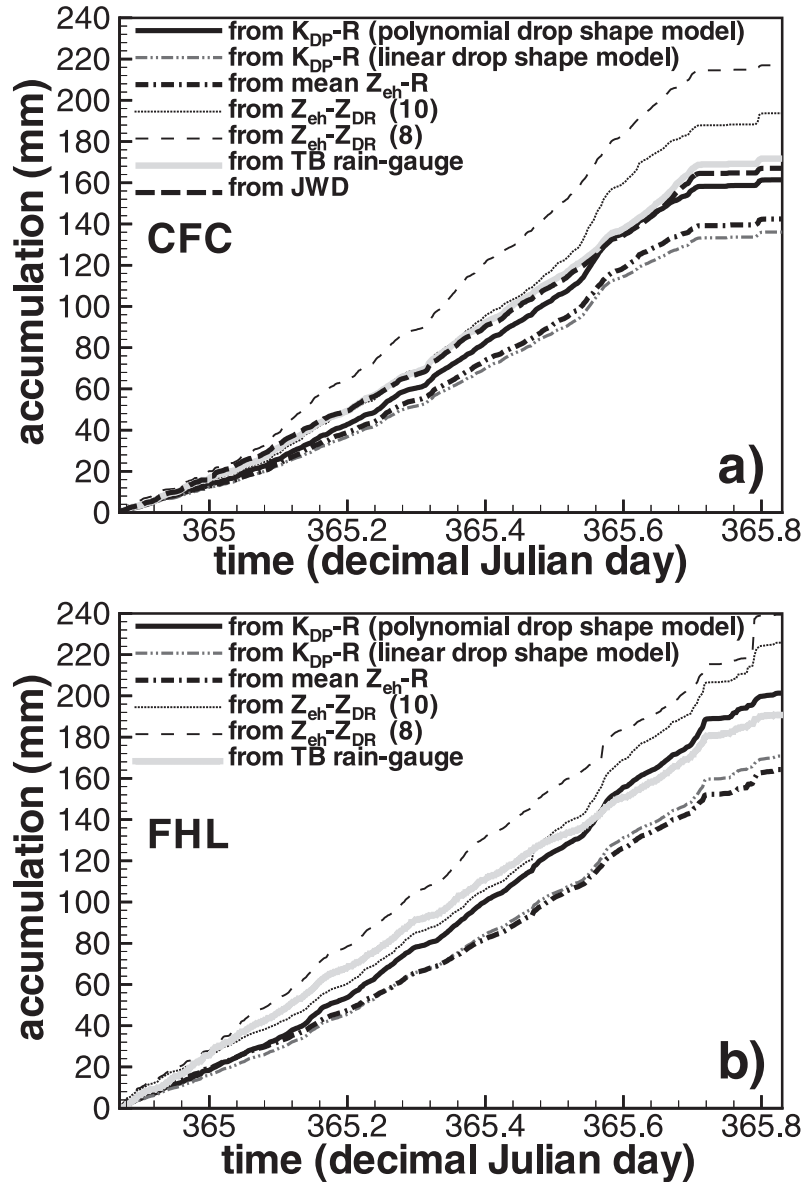

FIG. 7. Comparisons of rainfall accumulations from different radar estimators over the (a) CFC and (b) FHL ground validation sites with surface measurements for IOP 4 (30-31 Dec 2005).

located above these sites). The $Z_{\mathrm{eh}}$ and $Z_{\mathrm{DR}}$ measurements were corrected for attenuation/differential attenuation effects (Matrosov et al. 2005). The results for the mean $Z_{\mathrm{eh}}=100 R^{1.76}$ relation obtained using HMT06 DSDs (Matrosov et al. 2007) are also shown. Because of the noisiness of $K_{\mathrm{DP}}$ calculations in lighter rainfalls (e.g., Matrosov et al. 2006), rainfall rates from the mean $Z_{\mathrm{eh}}-R$ relation were used in the $K_{\mathrm{DP}}$-based estimators (for both drop shape models) if reflectivity in the corresponding range gate was less than $29 \mathrm{~dB} Z$.

It can be seen from Fig. 7 that, although there is a spread of about $\pm 30 \%$, the radar estimates over the ground sites generally track the surface-based measurements well. Accumulations from $Z_{\mathrm{eh}}-Z_{\mathrm{DR}}$-based relations are generally higher than surface data, whereas the $K_{\mathrm{DP}}$-based relation for the polynomial drop shape model results in the best agreement with these data. The agreement between the JWD and collocated TB rain gauge accumulations at the CFC site is very good, which provides additional confidence in JWD DSD measurements. 

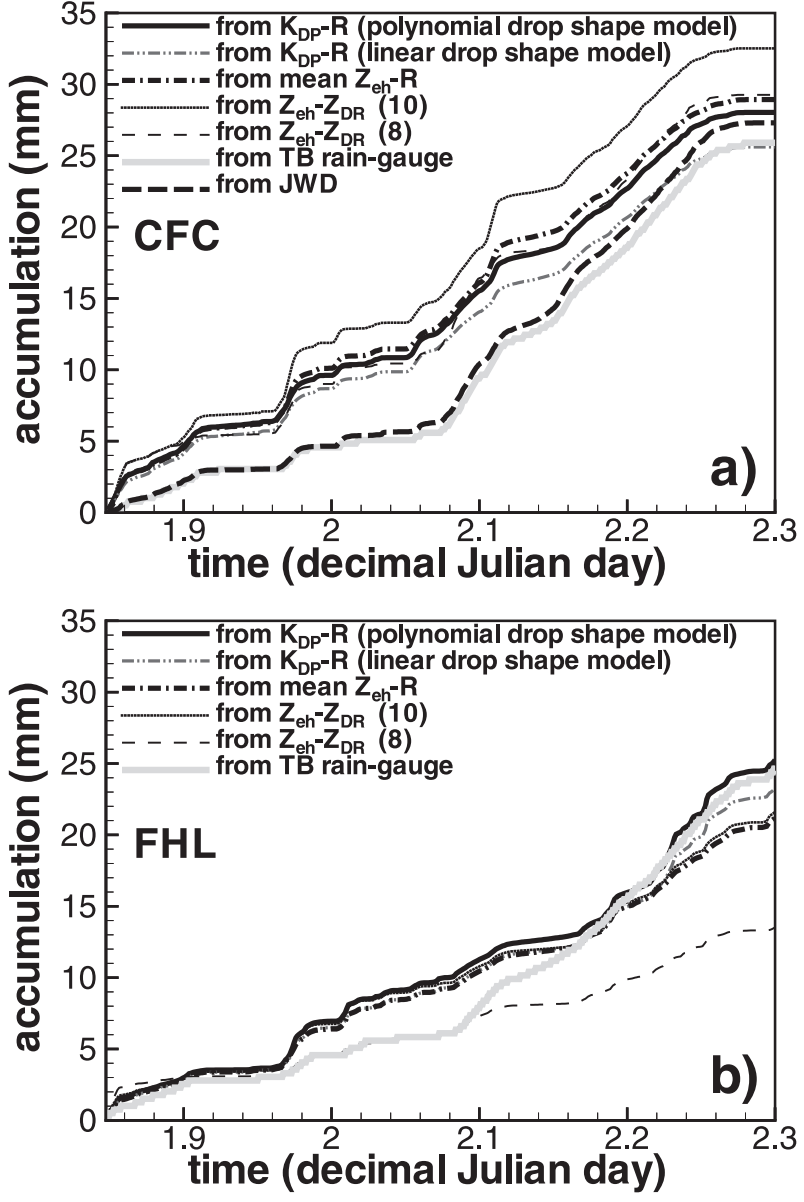

FIG. 8. As in Fig. 7, but for IOP 5 (2-3 Jan 2006).

Although IOP 4 was an extreme precipitation event and a few other IOPs experienced accumulations between about 80 and $160 \mathrm{~mm}$, some IOPs during the HMT-06 field project produced only about $20-25 \mathrm{~mm}$ of rainfall in comparable times. One such "lighter" event was observed during IOP 5 on 2-3 January 2006. Figure 8 presents rainfall accumulation time series for the warm part of this event, when the radar resolution volumes above $\mathrm{CFC}$ and FHL sites were in the rain region (i.e., below the melting layer). The radar estimates at the CFC site were somewhat higher than surface measurements, and the discrepancy was the largest in the first half of the comparison period. During this time, average rainfall was light, so radar estimators were mostly relying on reflectivity measurements. The variability in correspondence between reflectivity and rainfall is one possible explanation for this discrepancy.

Results of comparisons of radar-based estimates with surface gauge measurements, which were considered as the ground truth, for all HMT-06 IOPs (when the HYDROX radar was operational) and the sites, when
TABLE 1. Comparison results for radar-derived rainfall accumulations vs gauge estimates.

\begin{tabular}{lcc}
\hline \hline \multicolumn{1}{c}{ Estimator } & $\begin{array}{r}\text { Relative } \\
\text { bias }(\%)\end{array}$ & $\begin{array}{r}\text { Relative std } \\
\text { dev }(\%)\end{array}$ \\
\hline$Z_{e}=100 R^{1.76}$ & -8 & 23 \\
$R=17.0 K_{\mathrm{DP}}^{0.73}$ & 3 & 17 \\
$R=14.9 K_{\mathrm{DP}}^{0.79}$ & -9 & 22 \\
$R=17\left[Z_{\mathrm{eh}}\left(0.00012-0.000041 Z_{\mathrm{DR}}\right)\right]^{0.73}$ & 6 & 20 \\
$R=0.0039 Z_{\mathrm{eh}}^{1.07} Z_{\mathrm{dr}}^{-5.97}$ & -4 & 32 \\
$R=0.0056 Z_{\mathrm{eh}}^{1.02} Z_{\mathrm{dr}}^{-5.6}$ & -4 & 28 \\
\hline
\end{tabular}

the radar resolution volume above these sites was in the rain region are shown in Table 1. A zero mean and an $8^{\circ}$ standard deviation for drop canting was assumed and accounted for in radar estimates. The relative biases of radar estimates versus gauge data are relatively small. The $K_{\mathrm{DP}}$-based estimators (especially the one derived for the polynomial drops shape-size model) exhibit smallest relative standard deviations, which is likely due to the lower variability of these estimators to the DSD details. Note that these Table 1 standard deviations are smaller than those that characterize the scatter of the $R-K_{\mathrm{DP}}$ relations in Fig. 3 because of the partial cancelation of errors when rainfall accumulations are calculated from individual estimates of rain rate. The use of the considered $R-\left(Z_{\mathrm{eh}}-Z_{\mathrm{DR}}\right)$ relations provided similar results. The corresponding relative standard deviations for these relations were larger than for other estimators, which is, in part, due to a high sensitivity of these relations to differential reflectivity measurements (because of the exponent $\beta \sim-5.6$ to -6.0 ), which are usually more noisy than measurements of reflectivity.

\section{b. Comparisons of mass-weighted drop sizes}

Because $D_{m}$ retrievals are instantaneous estimates, the comparisons between the radar $Z_{\mathrm{DR}}$-based values with the CFC JWD data are subject to uncertainties caused by the radar-disdrometer sampling volume disparity and the spatial variability of rainfall in the vertical. In spite of these uncertainties, the comparison presents a certain interest. For IOP 4, Fig. 9 shows scatterplots between mass-weighted drop sizes estimated using differential reflectivity in the radar volume above the CFC site and the JWD estimates of $D_{m}$. Because the radar resolution volume was centered at about $800 \mathrm{~m}$ above the CFC site, $Z_{\mathrm{DR}}$-based estimates are compared to the surface data corresponding to a time 2 min later than the time of the radar measurements. This time difference is approximately required for drop populations to reach the ground at typically observed Doppler velocities. Because the uncertainties of $Z_{\mathrm{DR}}$ values are at least $0.2 \mathrm{~dB}$, the comparisons were performed only for those radar-based retrievals when differential reflectivity values (corrected 

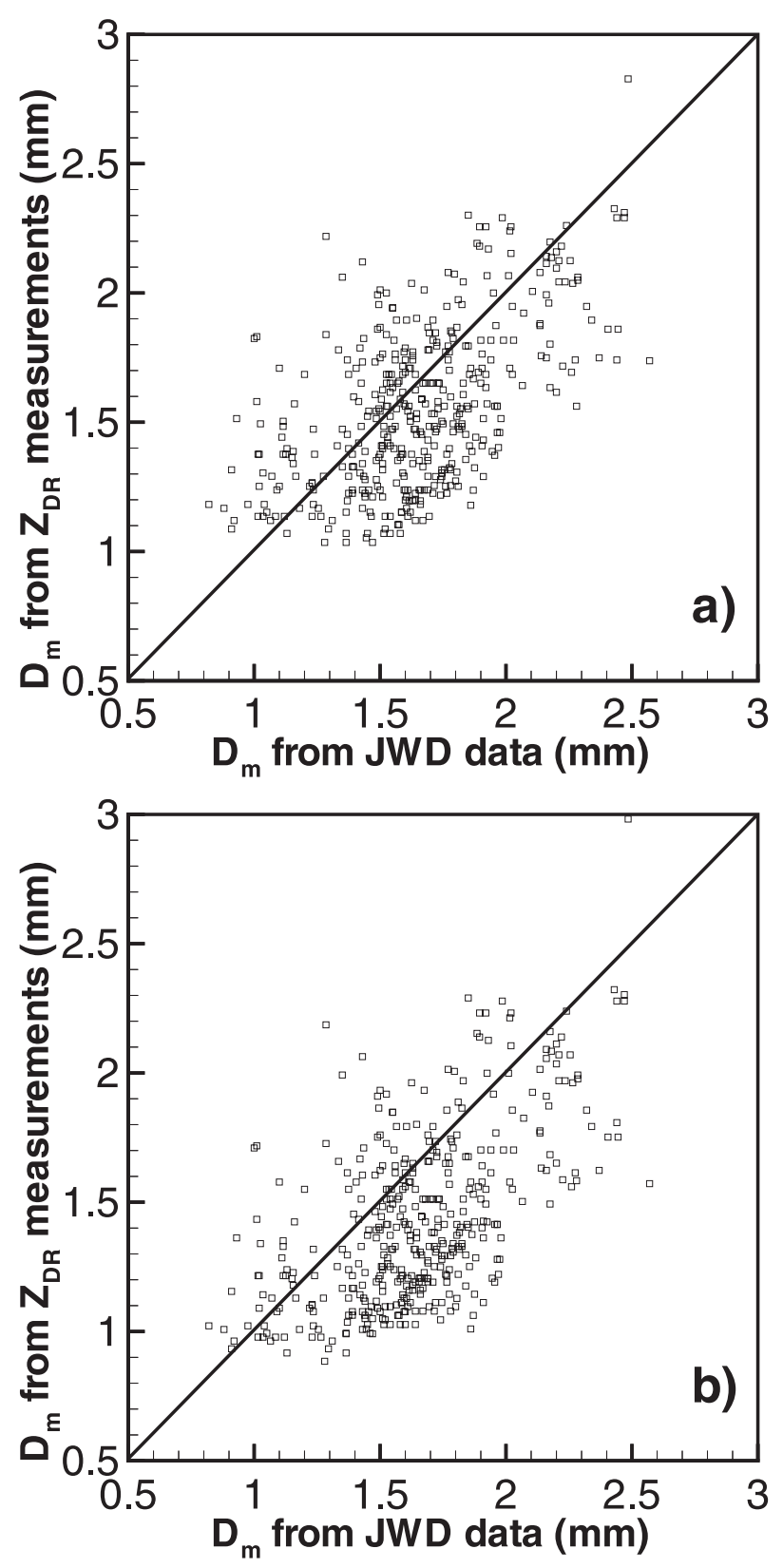

FIG. 9. Scatterplots of radar and surface disdrometer derived mean mass-weighted drop sizes assuming (a) polynomial and (b) linear drop shape models for IOP 4 (30-31 Dec 2005).

for differential attenuation) were greater than $0.2 \mathrm{~dB}$. This threshold corresponds to $D_{m} \approx 1 \mathrm{~mm}$ and $D_{m} \approx$ $0.75 \mathrm{~mm}$ for polynomial and linear drop shape models, respectively.

It can be seen from Fig. 9 that there is a general correspondence between JWD and radar-derived characteristic drop sizes. The correlation coefficient is about $r_{c}=0.6$ for both drop shape models, which reflects only a moderate correlation. The relative biases of radar estimates with respect to JWD data are about $-4 \%$ and $-12 \%$ for the polynomial and linear drop shape models, respectively. The relative standard deviations are about $20 \%$ and $24 \%$, respectively. For a fixed drop shape model, such standard deviation values are on the order of the $D_{m}$ retrieval uncertainty (considering the data scatter in Fig. 5 and assuming the 0.2-dB uncertainty in $Z_{\text {DR }}$ ). Although only IOP-4 data are shown in Fig. 9, similar biases and standard deviations were observed for other IOPs during HMT-06.

\section{The importance of the attenuation correction}

Radar signals at X-band frequencies are attenuated by rain that is noticeably stronger than at frequencies that are traditionally used in meteorological precipitation radars (i.e., S- and C-band frequencies). However, different rainfall estimators are affected by attenuation in their own way. Although the correction procedures for attenuation-differential attenuation effects using differential phase shift measurements with the HYDROX radar data are considered generally robust, it is instructive to estimate the influence of these effects on QPE.

For the extreme event observed during IOP 4, Fig. 10a depicts rainfall accumulation time series at the $\mathrm{CFC}$ ground validation site calculated for all the considered estimators using measured values of $Z_{\mathrm{eh}}$ and $Z_{\mathrm{DR}}$, which were not corrected for attenuation and differential attenuation. Comparing Figs. 7a and 10a shows that the $Z_{\mathrm{eh}}-R$ relation-based results diminish by about $40 \%$ as a result of ignoring attenuation effects. The corresponding QPE reduction for both $K_{\mathrm{DP}}$-based estimators is only around $10 \%$. This small reduction is caused by the fact that, for lighter rain periods with noisy differential phase data, rainfall rates from these estimators are calculated using the $Z_{\mathrm{eh}}-R$ relation. Such periods were not very significant for this IOP, so the corresponding reduction in $K_{\mathrm{DP}^{-}}$ based QPE due to ignoring attenuation is significantly less compared to the $Z_{\mathrm{eh}}-R$ relation results. Ignoring attenuation/differential attenuation when applying the $Z_{\mathrm{eh}}-Z_{\mathrm{DR}}$ QPE estimators provides approximately a $27 \%$ [for (8)] and 55\% [for (10)] decrease in accumulation values. The smaller sensitivity of the estimator (8) is explained by a partial cancelation of errors for the ratio $Z_{\mathrm{eh}}^{1.07} / Z_{\mathrm{dr}}^{5.97}$ when both numerator and denominator decrease as attenuation and differential attenuation increase.

Comparing the IOP-4 QPE calculated with and without accounting for attenuation and differential attenuation for the FHL site (not shown), located by about $40 \%$ farther from the radar than the CFC site (i.e., $25.7 \mathrm{~km}$ versus $18.3 \mathrm{~km}$ ), indicates further decreases of QPE estimates calculated when ignoring attenuation-differential attenuation corrections. These decreases amount to a 

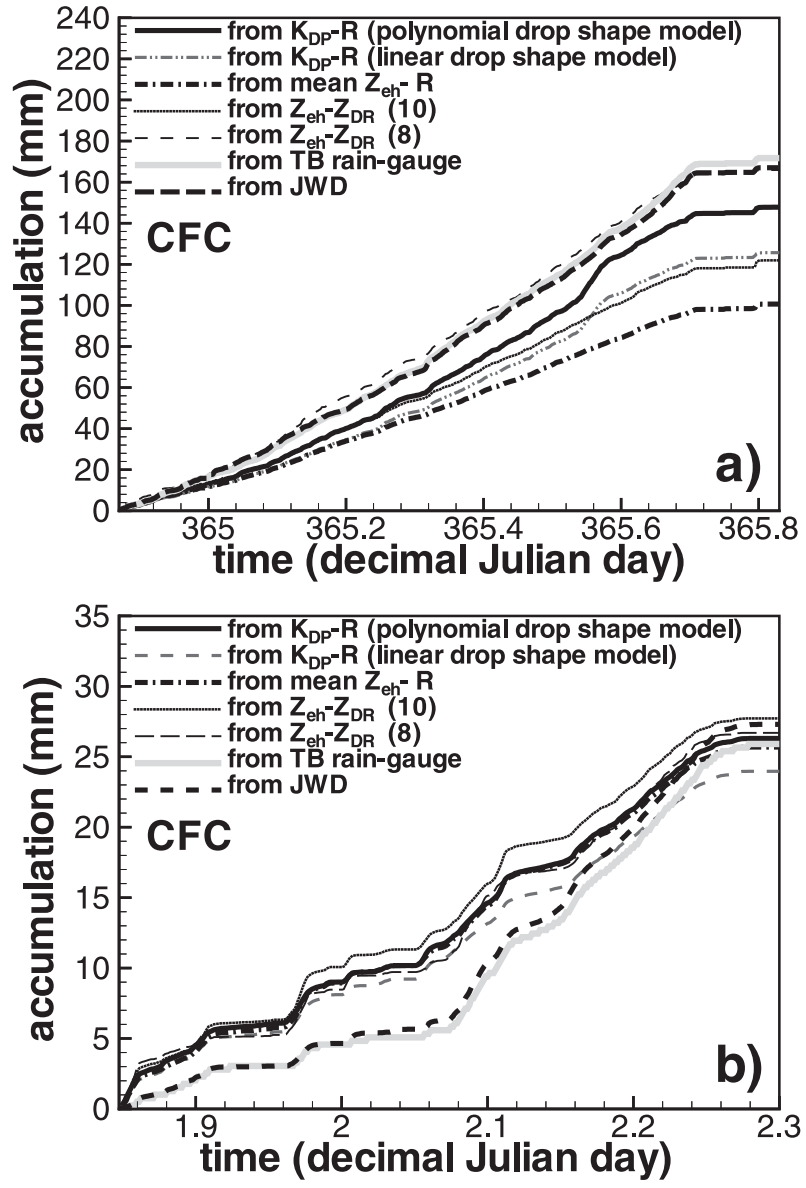

FIG. 10. Comparisons of rainfall accumulations from different radar estimators over the CFC site for (a) IOP 4 (30-31 Dec 2005) and (b) the warm part of IOP 5 (2-3 Jan 2006) when radar measurements were not corrected for attenuation-differential attenuation.

factor between 1.3 and 1.5 compared to percentage decreases for the CFC site. A factor of about 2 decrease could be expected at the distances that are close to the maximum range of the radar during the HMT-06 field project $(\sim 38.4 \mathrm{~km})$.

For a less intense rainfall as the one observed during the warm part of IOP 5, ignoring attenuation/differential attenuation corrections matters less. For this IOP, CFC site accumulations calculated with different estimators and ignoring these corrections are depicted in Fig. 10b. Comparing the results of this figure with the data from Fig. 8a, where corrections were accounted for, reveals about $12 \%$ (for the $Z_{\mathrm{eh}}-R$ relation) and $7 \%$ (for the $K_{\mathrm{DP}}$-based estimators) decreases in rainfall accumulation. The use of $Z_{\mathrm{eh}}-Z_{\mathrm{DR}}$-based QPE estimators exhibits the accumulation decreases of about $9 \%$ and $16 \%$ for relations (8) and (10), respectively.

The correction schemes for attenuation-differential attenuation effects are essential and necessary procedures for rainfall measurements with X-band polarimetric radars. However, certain uncertainties in the application of these procedures are possible, especially in real time during radar operations. The use of QPE estimators, which are less susceptible to these effects, may have some operational advantages.

\section{Summary and conclusions}

The data collected during the 4-month-long HMT-06 experiment, which was conducted in the foothills of California's Sierra Nevada, were used to evaluate different X-band radar-based estimators of rainfall parameters that use specific differential phase shift, differential reflectivity, and horizontal reflectivity measurements. The experimental raindrop size distributions and two different drop shape models were used for developing $\mathrm{X}$-band radar polarimetric estimators.

The results obtained with rainfall-rate radar estimators were then compared with the rainfall accumulation data from the surface sensors located in the radar coverage area. The $K_{\mathrm{DP}}$-based estimator, which assumes the drop aspect ratio described as a polynomial function of the drop size, provided the best agreement overall, with the surface data resulting in a small mean bias $(\sim 3 \%)$ and an approximately $17 \%$ standard deviation. An $R-K_{\mathrm{DP}}$ relation, assuming a mean linear change $(b \sim$ $0.56 \mathrm{~cm}^{-1}$ ) for drop aspect ratios as their size increases beyond $0.05 \mathrm{~cm}$, provides rainfall rates that are $15 \%-$ $20 \%$ smaller than those obtained assuming the mean polynomial drop shape. However, for a typical rainfall observed during HMT-06 (e.g., during IOP 5), the accumulation difference between the two $K_{\mathrm{DP}}$-based estimators is only about $10 \%$ because, for lighter rainfall rates, when differential phase measurements are too noisy, both estimators resort to the mean HMT- $06 Z_{\mathrm{eh}}-R$ relation. When this $Z_{\mathrm{eh}}-R$ relation was used exclusively, regardless of the observed reflectivity (and when reflectivity measurements were corrected for attenuation), it provided the accumulation results that were, on average, similar to those obtained with the $K_{\mathrm{DP}}$-based estimator, which assumes the mean linear drop shape. For a given drop shape assumption, the variability of the mean $R-K_{\mathrm{DP}}$ relation, depending on the origin of the DSD dataset, is smaller than that for the $Z_{\mathrm{eh}}-R$ relation. This is a consequence of the proportionality of $K_{\mathrm{DP}}$ and $R$ to more similar DSD moments than $Z_{\mathrm{eh}}$ and $R$.

As one might expect, the $Z_{\mathrm{eh}}-Z_{\mathrm{DR}}$ rainfall estimator (10), which is based on the consistency of $Z_{\mathrm{eh}}, Z_{\mathrm{DR}}$, and $K_{\mathrm{DP}}$ values and the polynomial model of the raindrop aspect ratio, provided accumulation results that are similar (in terms of the mean relative bias and standard deviation) to the data from the $K_{\mathrm{DP}}$ estimator based on 
the same drop shape model. However, the consistency $Z_{\mathrm{eh}}-Z_{\mathrm{DR}}$-based estimator, which was derived in this study for the polynomial drop shape-size model and for rainfalls that do not exhibit high values of $Z_{\mathrm{DR}}$ is rather sensitive to possible errors in the attenuation-differential attenuation corrections. These corrections depend on the raindrop aspect ratio assumption, and the difference between the mean polynomial and linear drop shape models can result in uncertainties of approximately 1.2 and $0.2 \mathrm{~dB}$ (for $Z_{\mathrm{eh}}$ and $Z_{\mathrm{DR}}$, respectively) at farther ranges of the $\mathrm{X}$-band radar coverage in case of heavier mean rainfall observed during HMT-06 (i.e., $\sim 9 \mathrm{~mm} \mathrm{~h}^{-1}$ ).

Power-law $Z_{\mathrm{eh}}-Z_{\mathrm{dr}}$ rainfall estimators expressed by a ratio of different powers of reflectivity and differential reflectivity $\left(R=c Z_{\mathrm{eh}}^{\alpha} Z_{\mathrm{dr}}^{\beta}\right)$ provide the highest standard deviation of the estimated accumulations with respect to the surface rain gauge data $(\sim 30 \%)$ because of their large values of the $Z_{\mathrm{dr}}$ exponent $(\beta \sim-5.6$ to -6$)$. However, one advantage of such estimators is their smaller susceptibility to the uncertainties in the attenuationdifferential attenuation corrections. Unlike the $R-K_{\mathrm{DP}}$ relations, $Z_{\mathrm{eh}}-Z_{\mathrm{DR}}$-based rainfall estimators provide greater coverage, because they are point estimators, whereas $K_{\mathrm{DP}}$ values are calculated as range derivatives of the differential phase shift measurements and require a certain path length for these calculations. These estimators are also less susceptible to the X-band backscatter resonances, which might contaminate $K_{\mathrm{DP}}$ values by differential backscatter phase shifts.

The similarity of polarimetric relations obtained with HMT-06 and HMT-07 DSDs suggests that the results presented in this study for the HMT-06 HYDROX radar deployment might be generally representative for the winter season precipitation in the American River basin. Although the suggested relations might be used directly in future HMT deployments for QPE purposes, one can also envision fine tuning these relations based on the availability of simultaneous DSD measurements in the radar coverage area.

Comparisons of the mean mass-weighted rain drop sizes retrieved from the $\mathrm{X}$-band radar $Z_{\mathrm{DR}}$ measurements with estimates from DSDs derived from JWD data showed a better agreement when using the mean polynomial drop shape assumption. The relative standard deviation between radar and surface estimates was about $20 \%-28 \%$, which is consistent with $Z_{\mathrm{DR}}$ uncertainties and data scatter resulting from the DSD variability. However, the correlation coefficient between radar and surface $D_{m}$ estimates is not very high $(\sim 0.6)$, which is due in part to vastly different sampling volumes. Uncertainties of differential reflectivity measurements are likely to prevent meaningful estimates of $D_{m}$ values that are smaller than about $1 \mathrm{~mm}$.
Acknowledgments. This research was funded by the NOAA Hydrometeorological Testbed Project. Many scientists and engineers from the ESRL Physical Science Division (including B. Martner, D. Kingsmill, E. Sukovich, K. Clark, K. King, and T. Ayers) were engaged in the deployment and servicing the radar and the surface meteorology instrumentation during the HMT-06 field project.

\section{REFERENCES}

Anagnostou, E. N., M. N. Anognostou, W. F. Krajewski, A. Kruger, and B. J. Mirovsky, 2004: High resolution rainfall estimation from X-band polarimetric radar measurements. J. Hydrometeor., 5, 110-128.

Andsager, K., K. V. Beard, and N. F. Laird, 1999: Laboratory measurements of axis ratios for large raindrops. J. Atmos. Sci., 56, 2673-2683.

Barber, P., and C. Yeh, 1975: Scattering of electromagnetic waves by arbitrarily shaped dielectric bodies. Appl. Opt., 14, 28642872.

Beard, K. V., and C. Chuang, 1987: A new model for equilibrium shape of rain drops. J. Atmos. Sci., 44, 1509-1524.

Brandes, E. A., G. Zhang, and J. Vivekanandan, 2005: Corrigendum. J. Appl. Meteor., 44, 186.

Bringi, V. N., and V. Chandrasekar, 2001: Polarimetric Doppler Weather Radar. Cambridge University Press, 636 pp.

Brotzge, J., K. Droegemeier, and D. McLaughlin, 2006: Collaborative adaptive sensing of the atmosphere (CASA): A new radar system for improving analysis and forecasting of surface weather conditions. Transp. Res. Rec., 1948, 145-151.

Campbell Scientific, 2008: Met One rain gage models 380 and 385. Campbell Scientific Instruction Manual, 22 pp. [Available online at http://www.campbellsci.com/documents/manuals/met1. pdf.]

Doviak, R. J., V. Bringi, A. Ryzhkov, A. Zahrai, and D. Zrnic, 2000: Considerations for polarimetric upgrades to operational WSR-88D radars. J. Atmos. Oceanic Technol., 17, 257-278.

Goddard, J. W. F., J. Tan, and M. Thurai, 1994: Technique for calibration of meteorological radar using differential phase. Electron. Lett., 30, 166-167.

Gorgucci, E., G. Scarchilli, and V. Chandrasekar, 1992: Calibration of radars using polarimetric techniques. IEEE Trans. Geosci. Remote Sens., 30, 853-858.

,,--- , and V. N. Bringi, 2000: Measurement of mean raindrop shape from polarimetric radar observations. $J$. Atmos. Sci., 57, 3406-3413.

_- L. Baldini, and V. Chandrasekar, 2006: What is the shape of a raindrop? An answer from radar measurements. J. Atmos. Sci., 63, 3033-3044.

Gunn, R., and G. D. Kinzer, 1949: The terminal velocity of fall for water droplets in stagnant air. J. Meteor., 6, 243-248.

Joss, J., and A. Waldvogel, 1967: Ein Spektrograph für Niederschlagstropfen mit automatisher Auswertung. Pure Appl. Geophys., 68, 240-246.

Matrosov, S. Y., 2004: Depolarization estimates from linear H and $\mathrm{V}$ measurements with weather radars operating in simultaneous transmission-simultaneous receiving mode. J. Atmos. Oceanic Technol., 21, 574-583.

, K. A. Clark, B. E. Martner, and A. Tokay, 2002: X-band polarimetric radar measurements of rainfall. J. Appl. Meteor., 41, 941-952. 
— D. E. Kingsmill, and F. M. Ralph, 2005: The utility of X-band polarimetric radar for quantitative estimates of rainfall parameters. J. Hydrometeor., 6, 248-262.

—, R. Cifelli, P. C. Kennedy, S. W. Nesbitt, S. A. Rutledge, V. N. Bringi, and B. E. Martner, 2006: A comparative study of rainfall retrievals based on specific differential phase shifts at $\mathrm{X}$ - and S-band radar frequencies. J. Atmos. Oceanic Technol., 23, 952-963.

- C. A. Clark, and D. A. Kingsmill, 2007: A polarimetric radar approach to identify rain, melting-layer, and snow regions for applying corrections to vertical profiles of reflectivity. J. Appl. Meteor. Climatol., 46, 154-166.

Park, S.-G., M. Maki, K. Iwanami, V. N. Bringi, and V. Chandrasekar, 2005: Correction of radar reflectivity and differential re- flectivity for rain attenuation at $\mathrm{X}$ band. Part II: Evaluation and application. J. Atmos. Oceanic Technol., 22, $1633-1655$.

Pruppacher, H. R., and K. V. Beard, 1970: A wind tunnel investigation of the internal circulation and shape of water drops falling at terminal velocity in air. Quart. J. Roy. Meteor. Soc., 96, 247-256.

Sheppard, B. E., and P. I. Joe, 1994: Comparisons of raindrop size distribution measurements by a Joss-Waldvogel disdrometer, a PMS 2DG spectrometer, and a POSS Doppler radar. J. Atmos. Oceanic Technol., 11, 874-887.

Thurai, M., and V. N. Bringi, 2005: Drop axis ratios from a 2D video disdrometer. J. Atmos. Oceanic Technol., 22, 966-978. 